\title{
Vom Rand in die Mitte - „Schamlose Normalisierung“
}

\author{
Ruth Wodak
}

Online publiziert: 18 . April 2018

(C) Der/die Autor(en) 2018

Zusammenfassung Dieser Beitrag diskutiert den offensichtlichen Rechtsruck der (österreichischen) Parteienlandschaft, der in den Nationalratswahlen am 15. Oktober 2017 verdeutlicht wurde. Ein Prozess der Normalisierung, so die Annahme, bedingt eine Anpassung an die, teilweise rechtsextremen, Agenden rechtspopulistischer Parteien und führt zu einer ausschließlichen Fokussierung von Migrations- und Flüchtlingspolitik. Nach dem Versuch, das Phänomen „Rechtspopulismus“ klar zu definieren, wird die schamlose Enttabuisierung vormals tabuisierter rechtsextremer Inhalte und Äußerungen am Beispiel der Annäherung der neuen Österreichischen Volkspartei (ÖVP) an die Freiheitliche Partei Österreichs (FPÖ) im Detail illustriert.

Schlüsselwörter Normalisierung $\cdot$ Rechtspopulismus $\cdot$ Diskursforschung · Ideologie · Rechtsextremismus · „Post-Scham“

\section{Transforming Marginalised Politics into Mainstream Agenda-"Shameless Normalisation"}

\begin{abstract}
In this paper, I discuss the swing to the (far-)right in (Austrian) party politics during the election campaign and national election on October 15, 2017. This transformation is caused, I claim, by a process of normalisation, an accommodation to the, sometimes also extreme-right, agenda of formerly right-wing populist parties such as the Austrian Freedom Party (FPÖ). The election campaign of both the Austrian People's Party (ÖVP) and the FPÖ focussed primarily on migration and refugee politics, based on a politics of fear and resentment. After first attempting to
\end{abstract}

R. Wodak $(\triangle)$

Lancaster, Vereinigtes Königreich

E-Mail: r.wodak@lancaster.ac.uk

R. Wodak

Wien, Österreich 
define the phenomenon of right-wing populism, I trace how tabooed and extreme right contents slowly became acceptable, as soon as the ÖVP shamelessly integrated some (not all) aspects of the FPÖ's election program.

Keywords Normalisation · Right-wing populism · Extreme-right · Discourse studies · Ideology • "Post-shame"

\section{Normalisierung nach rechts}

In diesem Beitrag diskutiere ich den offensichtlichen Rechtsruck in der (österreichischen) Parteienlandschaft, die Enttabuisierung und Akzeptanz früher tabuisierter Inhalte wie Äußerungsformen. Dies bezeichne ich als „Normalisierung“. Eine solche Normalisierung geht, wie zu beobachten ist, mit einer gewissen „Schamlosigkeit“" vor sich: Demnach verschieben sich einerseits die Grenzen des Sagbaren, sowohl, was die Häufigkeit von Unwahrheiten und das Infragestellen und Brechen von Diskurskonventionen betrifft, als auch in Bezug zu wiederholten Angriffen auf zentrale demokratische Institutionen, wie Medien und Justiz. Zunächst wird kurz beschrieben, in welcher Weise sich die konservative Österreichische Volkspartei (ÖVP) zu einer rechtspopulistischen Partei normalisiert (hat) und Themen wie Vorschläge der rechtsextremen Freiheitlichen Partei Österreichs (FPÖ) übernimmt; danach präsentiere ich eine Arbeitsdefinition des Begriffs „Rechtspopulismus“. Im dritten Abschnitt mögen einige Beispiele aus der österreichischen Frontstage- und BackstagePolitik 2017/18 die behauptete Verflechtung von Normalisierung und Schamlosigkeit belegen.

Ausgehend von Jürgen Links (2013) Überlegungen zum Konzept des Normalismus wandten sich Margaret und Siegfried Jäger (2007) den Verschiebungen innerhalb einer politischen Landschaft durch die „Normalisierung des Nicht-Normalen“ zu. Insbesondere die Normalisierung der Bewertung von MigrantInnen als Bedrohung der inneren Sicherheit und Belastung des Sozialstaats und Bildungswesens ist als länderübergreifende Entwicklung zu sehen (Wodak 2015a, 2015b; Rheindorf 2017).

Auch bei den österreichischen Nationalratswahlen am 15. Oktober 2017 fand eine solche Normalisierung statt: So fokussierte die ÖVP (nunmehr „Liste Sebastian Kurz - Die neue Volkspartei“, von schwarz zu türkis umgefärbt) fast ausschließlich das Migrationsthema (wobei Flüchtlinge mit sogenannten ,illegalen Migranten“ gleichgesetzt wurden ${ }^{1}$ ), versprach, die „Mittelmeerroute“ zu sperren, die gesetzlich festgelegte Mindestsicherung (für anerkannte Flüchtlinge aber auch andere Bedürftige) zu verringern und insgesamt die seit 2016 beschlossene Obergrenze von 37.500 Asylwerbern gegen Null zu senken (obwohl die Zahl der Neuankommenden seit

\footnotetext{
1 Vergleiche den dritten Teil des ÖVP Wahlprogramms zu Ordnung und Sicherheit: https://secure. sebastian-kurz.at/ordnung-und-sicherheit/\&usg=ALkJrhin9CszbrB0sNM3hlNFAoppXPMGqwRegierun gsprogramm. Zugegriffen: 10.03.2018.
} 
2015 wesentlich niedriger geworden ist) ${ }^{2}$. Damit übernahm der seit 1 . Juli 2017 neu installierte Parteiobmann fast wortwörtlich die Programmatik der - wie ich behaupte - rechtsextremen FPÖ.

Wie Hans-Hennig Scharsach (2017) in seinem Buch Stille Machtergreifung eindeutig nachweist, haben sich die Strukturen der FPÖ seit 2005 unter dem Vorsitz von Heinz-Christian (HC) Strache signifikant verändert, und das immer mehr in eine rechtsextreme Richtung ${ }^{3}$ : Schlagende Burschenschafter, die insgesamt nur 0,4\% der österreichischen Bevölkerung ausmachen, haben, wie Hans Rauscher pointiert feststellt,

„de facto die FPÖ übernommen. [...]. Strache, Hofer, Stefan, Gudenus und Haimbuchner bilden den Bundesvorstand der FPÖ, also das höchste Führungsgremium. Sie gehören alle zu schlagenden studentischen Verbindungen oder „Burschenschaften“ [...]. Die Führungsspitze der FPÖ besteht zu $100 \%$ aus „Alemannen“, „Marko-Germanen“ und „Vandalen““” (Rauscher 2017). ${ }^{4}$

Wesentliche Charakteristika des Rechtsextremismus, wie das Befürworten von Antiliberalismus, autoritärem Führer- und Gefolgschaftsprinzip, einer sogenannten „Volksgemeinschaft“, von Antifeminismus und Rassismus, treffen, so Rauscher, auf die meisten Burschenschaften zu.

Das Schüren von Ressentiments und Ängsten durch Kurz und Strache war demnach erfolgreich. ${ }^{5}$ Sowohl die neuerdings national-konservative (rechtspopulistische) ÖVP gewann 4,7\% seit 2013 dazu und belegte mit 31,5\% den ersten Platz als auch die dritt-platzierte FPÖ legte mit $26 \%$ um 5,5\% zu. ${ }^{6}$ Aufgrund der großen programmatischen Ähnlichkeiten zwischen FPÖ und ÖVP wurden alsbald Koalitionsgespräche begonnen. Die neue türkis-blaue Regierung wurde, begleitet von lautstarken Protesten, am 18. Dezember 2017 von Bundespräsident Alexander Van Der Bellen angelobt. ${ }^{7}$ Der ÖVP unter ihrem neuen Obmann ist es also gelungen, die Kanzlerschaft zu erringen. Aber um welchen Preis? ${ }^{8}$

\footnotetext{
2 Vergleiche dazu https://de.statista.com/statistik/daten/studie/293189/umfrage/asylantraege-in-oester reich/. Zugegriffen: 30.11.2017.

3 Vergleiche Pfahl-Traughber (2015, S. 75-81) zu den Unterschieden zwischen Rechts-, Links- und religiösem Extremismus.

4 Zur Geschichte und Entwicklung der FPÖ siehe Manoschek (2002), Rheindorf und Wodak (2018), sowie Wodak und Rheindorf (2018).

5 An dieser Stelle ist es unmöglich, den komplexen und von vielen (Medien)Skandalen, Pannen, Gerüchten und teilweise kriminellen Machenschaften gezeichneten Wahlkampf im Detail zu beschreiben. Vielmehr geht es hier darum, den Wandel des hegemonialen Diskurses und akzeptierter Praxen aufgrund einer ,erfolgreichen“ Anpassung und Übernahme rechtspopulistischer/rechtsextremer Inhalte wie Rhetorik nachzuvollziehen.

6 Vergleiche zu Einzelheiten: http://www.spiegel.de/politik/ausland/oesterreich-alle-ergebnisse-dernationalratswahl-2017-a-1172061.html. Zugegriffen: 30.11.2017.

7 Vergleiche dazu: https://derstandard.at/2000070495198/regierungsprogramm-oevp-fpoe-kurz-stracheueberblick-analyse. Zugegriffen: 04.03.2018. Und: https://derstandard.at/jetzt/livebericht/2000070552695/ koalition-liveticker-neue-oevp-fpoe-regierung-angelobt-tausende-bei-protesten-in-wien. Zugegriffen: 04.03.2018.

8 Siehe dazu: http://www.sueddeutsche.de/politik/seite-drei-ueber-den-wahlausgang-in-oesterreichkuess-die-hand-1.3710650?reduced=true. Zugegriffen: 30.11.2017.
} 
Die Übernahme rechtspopulistischer Agenden durch die ÖVP impliziert einen deutlichen Rechtsruck (ähnliche Entwicklungen kann man in anderen EU-Mitgliedstaaten verfolgen), eine Aufgabe integraler christlich-sozialer Werte und einer bisher eindeutig pro-europäischen Haltung. Dass manche FPÖ-Politiker die allgemein gültigen Menschenrechte infrage stellen, scheint nicht zu stören ${ }^{9}$. Anders als im Jahre 2000, als die erste ,schwarz-blaue“ Koalition in Österreich national und international auf vehemente Kritik stieß und in der Folge EU-weite Sanktionen gegen diese Regierung erlassen wurden (Wodak und Pelinka 2002), wurde und wird diesmal vor allem vonseiten der Zivilgesellschaft Unmut über den Einzug von 21 Burschenschaftlern als FPÖ-Parlamentarier und die Besetzung von Ministerposten mit rechtsextremen PolitikerInnen laut. Der am 26. Januar 2017 neu angelobte Bundespräsident Alexander Van der Bellen hatte im Vorfeld der Koalitionsverhandlungen einige FPÖ Mitglieder als nicht ministrabel bezeichnet (nämlich den Wiener Vize-Bürgermeister (ohne Portefeuille) Johann Gudenus und den Europaabgeordneten Harald Vilimsky) und sich erfolgreich gegen die Besetzung von Innen- und Justizministerium durch FPÖ-Minister gewehrt; er hatte auch den Transfer der EU-Agenda aus dem nun von der FPÖ geführten Außenministerium ins Bundeskanzleramt zu Kanzler Kurz durchgesetzt. Die türkis-blaue Koalition gelobte Van der Bellen dennoch an, trotz häufiger gegenteiliger Versicherungen während seiner Präsidentschaftswahlkampagne. ${ }^{10}$

\section{Was ist „Rechtspopulismus“"?}

Auf die Frage, was Rechtspopulismus ,ist“, gibt es keine einfache und eindeutige Antwort. Vielmehr zeigt die Menge an jüngst erschienenen Monografien, Sammelbänden, Buchkapiteln und Zeitschriftenartikeln, dass sich Wissenschaftlerinnen und Wissenschaftler vieler Disziplinen nicht einig sind: sowohl darüber, ob es das Phänomen „Rechtspopulismus“ überhaupt gibt, als auch darüber, wie man es definieren sollte, gerade in Abgrenzung zu anderen Ideologien und sozialen Bewegungen, wie Rechtsextremismus, ,alt-right“, Populismus, Faschismus und Linkspopulismus. Und darüber hinaus: Ist Rechtspopulismus eine Ideologie (thin bzw. thick; Kriesi und Pappas 2015, S. 5), eine Philosophie (Priester 2007, S. 9), ein spezifisches Medienphänomen (Pajnik und Sauer 2017) oder ein politischer Stil (Moffitt 2017), der sich v.a. performativ und kommunikativ äußert?

\footnotetext{
9 Vergleiche die Fülle von Kommentaren in nationalen und internationalen Zeitungen: https://www.welt. de/politik/ausland/article169657614/Klarer-Rechtsruck-in-Oesterreich-Kurz-mit-OEVP-vorne.html; https://www.zdf.de/nachrichten/heute/konservative-oevp- gewinnt-wahl-in-oesterreich-rechtsruck-zu100.html; https://www.stern.de/news/rechtsruck-in-oesterreich-nach-wahlsieg-der-oevp-und-starkem-ab schneiden-der-fpoe-7661374.html; https://www.nytimes.com/interactive/2016/05/22/world/europe/europeright-wing-austria-hungary.html?_r=0. Alle zugegriffen: 17.10.2017.

10 http://www.bundespraesident.at/aufgaben/aufgaben-und-rechte/. Zugegriffen: 30.11.2017. Zu den voreiligen Aussprüchen Van der Bellens in Bezug auf eine Regierungsbeteiligung der FPÖ vergleiche: https:// diepresse.com/home/politik/innenpolitik/4828916/Van-der-Bellen_Wuerde-FPOegefuehrte-Regierungnicht-angeloben. Zugegriffen: 04.03.2018.
} 
Im Vorwort zum neuen Handbook of the Radical Right (2017, S. 1-2) behauptet Jens Rydgren, dass der Begriff „Rechtspopulismus“ obsolet sei; vielmehr handele es sich um ,ethno-nationalistische“ Parteien, die allesamt auch ein populistisches Element besitzen:

Radical right parties and movements share an emphasis on ethno-nationalism rooted in myths about the past. Their programs are directed toward strengthening the nation by making it more ethnically homogenous and - for most radical right-wing parties and movements - by returning to traditional values. They also tend to be populists, accusing elites of putting internationalism ahead of the nation and of putting their own narrow self-interest and various special interests ahead of the interests of the people. (Rydgren 2017)

Im Gegensatz zu solchen ethno-nationalistischen Parteien seien rechtsextreme Parteien durch die Ablehnung des demokratischen Systems und dessen Institutionen charakterisiert. Allerdings, so Rydgren, verschwimmen manchmal die Grenzen zwischen ethno-nationalistischen und rechtsextremen Parteien. In eine ähnliche Kerbe schlägt Benjamin van de Cleen (2017, S. 8).

Im krassen Gegensatz zu diesem Ansatz positioniert sich Rogers Brubaker (2017), der Populismus als ,,a discursive and stylistic repertoire“ begreift. Er fokussiert die diskursiven, rhetorischen und stilistischen Gemeinsamkeiten, die seiner Meinung nach alle populistischen Bewegungen und Parteien charakterisierten. Dies sei Teil eines größeren, umfassenden ,discursive and stylistic turn“ (Brubaker 2017, S. 3). Zwischen diesen zwei Extremen begegnen wir anderen Definitionen, auf die ich weiter unten eingehen werde. Als Diskursforscherin ist es mir jedoch - im Gegensatz zu Brubaker - wichtig zu betonen, dass Rechtspopulismus nicht nur als rhetorischer Stil bzw. als ein reines mediales Performanzphänomen zu betrachten ist (wobei natürlich die [mediale] Inszenierung nicht zu unterschätzen ist; Januschek und Reisigl 2014), sondern dass die jeweils kommunizierten ideologischen Inhalte entscheidend sind. In der Tat wäre es, so der Kulturwissenschaftler Dick Pels (2012, S. 32), „falsch zu denken, es gäbe keine Substanz hinter diesem politischen Stil. [...] Gerade die dynamische Mischung aus Inhalt und Form hat der populistischen Politik in der heutigen Mediendemokratie eine führende Position bei den Wählern verschafft".

Verfolgt man die vielen Debatten in der Populismusforschung, so ist man mit heftigen Auseinandersetzungen konfrontiert - Populismus bzw. populistisch als negativer, gar inflationärer und vager alltagssprachlicher Begriff; oder als notwendiges demokratie-stärkendes Element (Laclau 2005), oder als demokratie-bedrohliche und demokratie-schädliche Agenda (Biskamp 2017; Grabbe und Lehner 2017). Weitere Auseinandersetzungen werden über den Appeal solcher Parteien geführt: Wen sprechen die Programme, Poster, Parolen und Slogans, die vielen verschiedenen, über traditionelle Medien wie auch über soziale Medien verbreiteten Textsorten der Kampagnen rechtspopulistischer Parteien an: v. a. Männer oder auch Frauen? Stammen die WählerInnen v. a. aus der Mittelschicht (wie von manchen behauptet wird) oder kommen sie aus allen sozialen Milieus und Altersgruppen (wie von anderen behauptet wird) oder doch eher aus den weniger gebildeten Schichten, also aus dem 
Arbeitermilieu (SORA 2017 ${ }^{11}$ )? In diesem Zusammenhang stellt sich offensichtlich die Frage, ob die traditionellen politischen Verortungen wie ,links“ bzw. ,rechts“ heutzutage überholt sind (Krasteva 2017).

Außerdem: Gibt es historische und kontext-bedingte Unterschiede, etwa zwischen den rechtspopulistischen Parteien der ehemaligen Ostblockländer und Westeuropas, zwischen dem reichen Norden und dem armen Süden, zwischen Ländern mit kolonialer und/oder faschistischer Vergangenheit und solchen ohne diese Entwicklungen, zwischen den kleinen Staaten, wie der Schweiz, Dänemark und Österreich, und den großen, wie Deutschland oder Frankreich? ${ }^{12}$ Es gibt natürlich noch weitere relevante Fragen, die ich aus Platzgründen nicht alle anführen kann (Stavrakakis 2017).

Zurück zu einer möglichen Definition von Rechtspopulismus: Es handelt sich, so Cas Mudde und Cristobal Rovira Kaltwasser, um eine (dünne) Ideologie, realisiert in unterschiedlichen diskursiven und materiellen Praxen. Mudde und Kaltwasser (2017, S. 9-12) heben drei Parameter hervor: erstens, die Opposition zwischen einem „Volk“ und den ,korrupten Eliten“. Zweitens, eine Fundierung in der volonté générale des Volkes. Drittens, eine dünne Ideologie, weil diese kein kohärentes Glaubensgebäude (Wodak 2017) abgibt, sondern in eklektischer Art widersprüchlichste Ideologeme versammelt. Da Mudde und Kaltwasser ihre Definition nicht auf den Rechtspopulismus beschränken, erfasst der Volks-Begriff sowohl das Volk als Souverän wie auch das gemeine (common) Volk. Außerdem kann das Volk als ethnos gemeint sein. Ebenso wird beim Konzept der Eliten differenziert, wobei die Eliten sowohl aufgrund (kultureller, ökonomischer und sozialer) Macht wie auch aufgrund ethnischer Kriterien erfasst werden können. Und schließlich gilt volonté générale als der allgemeine Wille des Volkes, ganz im Sinne Jean-Jacques Rousseaus.

Auf den Rechtspopulismus bezogen, muss nun diese recht allgemeine Definition in Hinblick auf mehrere Aspekte differenziert und ergänzt werden (Wodak 2015a, S. 20-22, S. 25-33). Vier Dimensionen sind meiner Meinung nach entscheidend:

- Nationalismus/Nativismus/Anti-Pluralismus: Rechtspopulistische Parteien beziehen sich auf ein scheinbar homogenes Ethnos, ein Populum (Gemeinschaft, Volk), das beliebig - häufig nach nativistischen (blutbezogenen) Kriterien - definiert wird; wer also die „wahren“ Österreicher, Deutschen, Ungarn oder Finnen sind, kann immer wieder neu bestimmt werden. Diese Parteien legen Wert auf ein Kernland (oder Heimat), das vor scheinbar gefährlichen Eindringlingen geschützt werden muss. Auf diese Weise werden Bedrohungsszenarien aufgebaut - die Heimat oder das „Wir“ werden von „Anderen“ bedroht: Fremde innerhalb der Gesellschaft und/oder von außerhalb, Migranten, Flüchtlinge, Türken, Juden, Roma, Banker, Moslems usw.

- Anti-Establishment/Anti-Elitismus: Diese Parteien teilen eine anti-elitäre und anti-intellektuelle Haltung (Arroganz der Ignoranz), verbunden mit starker EuroSkepsis. Außerdem werden plebiszitäre Verfahren bevorzugt, die Suche nach ei-

11 Vergleiche dazu: http://www.sora.at/themen/wahlverhalten/wahlanalysen/nrw17.html. Zugegriffen: 30.11.2017.

12 Siehe dazu ausführlich Betz (2017), Judt (2007), Krastev (2017), Pelinka (2013), und Wodak (2015a, 2016). 
ner „wahren Demokratie“, wobei eine sogenannte „formalistische Demokratie“ als Antonym dargestellt wird. Nach Ansicht dieser Parteien sollte die Demokratie auf das Mehrheitsprinzip des (jeweils willkürlich definierten) „Volkes“ reduziert werden.

- Autoritarismus: Ein Retter, ein charismatischer Führer, wird verehrt, der zwischen den Rollen von Robin Hood (Schutz des Sozialstaats, Unterstützung von „Mann und Frau auf der Straße“) und „strengem Vater" wechselt (Lakoff 2004). Solche charismatische Führerinnen und Führer benötigen eine hierarchisch organisierte Partei und autoritäre Strukturen, um Recht und Ordnung zu schaffen und für Sicherheit zu sorgen.

- Konservativismus/Geschichtsrevisionismus: Rechtspopulistische Parteien vertreten traditionelle, konservative Werte (traditionelle Geschlechterrollen und Familienwerte) und beharren auf dem Status quo bzw. sind rückwärtsgewandt. Der Schutz der Heimat bedingt auch den Glauben an ein gemeinsames Narrativ der Vergangenheit, in der ,Wir“ entweder Helden oder Opfer des Bösen waren (einer Verschwörung, von Feinden des Vaterlandes usw.). Damit mutieren vergangenes Leid oder Niederlagen zu Erfolgsgeschichten des Volkes oder zu Geschichten von Betrug und Verrat anderer. Sozialleistungen sollen im Sinne eines Wohlfahrtschauvinismus nur für die echten/wahren Mitglieder des ethnos gelten.

Obwohl nicht alle rechtspopulistischen Parteien alle Inhalte befürworten, können diese in jeweils bestimmter Kombination weitgehend verallgemeinert werden, als typisch für rechtspopulistische Ideologien. Durchgängig werben solche Parteien für Veränderung, weg von einem - so wird unterstellt - höchst gefährlichen Weg, der geradewegs in ein apokalyptisch ausgemaltes Inferno führe.

Angstmache bestimmt dementsprechend als durchgängige politisch-persuasive Strategie und als übergeordnetes Argumentationsmuster die Kampagnen rechtspopulistischer Parteien. Willkürlich werden Verantwortliche für die jeweils in einem ersten Schritt definierte Misere bestimmt - spezifische Sündenböcke. So dienen manchmal Juden, manchmal Muslime, Roma oder andere Minderheiten als Sündenböcke, manchmal Kapitalisten, Sozialisten, Karrierefrauen, Nicht-RegierungsOrganisationen (NGOs), die EU, die Vereinten Nationen, die USA oder Kommunisten, die Regierungsparteien, die Eliten, die Medien und so weiter. In einem dritten Schritt taucht nun der Retter aus der Not auf: der oder die jeweilige Parteivorsitzende, bereit, die Probleme auf einfache Weise zu „lösen“, etwa durch ein Schließen von Grenzen, Abschieben sogenannter illegaler Migranten usw. Ein neues, positives Narrativ wird angeboten, das Hoffnung wecken soll, im Gegensatz zur befürchteten Apokalypse. Die neue Vision, meist als unspezifizierte Veränderung beworben, ist jedoch rückwärtsgewandt, fußend auf einer längst überholten, anachronistischen Sehnsucht nach einer ethnisch homogenen, patriarchalen Gemeinschaft.

Ein weiteres Charakteristikum rechtspopulistischer Parteien muss noch betont werden, das die Performance in der Öffentlichkeit und die Medienpolitik bestimmt: Anhand ständiger Provokationen wird Aufmerksamkeit auf die jeweilige Führerpersönlichkeit und die politischen Agenden gelenkt (Wodak 2016, S. 38-40). Einerseits spielen „bad manners“ (Moffitt 2017, S. 61-63; Montgomery 2017, S. 632; Wodak 2017, S. 559-560) eine Rolle, bewusste Unhöflichkeiten, Unwahrheiten, Be- 
leidigungen, destruktive (eristische) Argumentation und intentionale Tabubrüche. Normen politischer Korrektheit werden verletzt, ohne sich dafür zu entschuldigen oder zu schämen (Scheff 2000); damit wird Identifikation mit anti-elitären Verhalten geboten. In diesem Zusammenhang sind auch Sagbarkeits- bzw. Möglichkeitsbedingungen von Interesse, denn diese konstituieren, was in einem bestimmten Diskurs sagbar ist, ohne dass die SprecherInnen dafür zur Rechenschaft gezogen werden bzw. ihr „Gesicht verlieren“ (Bettinger 2007, S. 77; Goffman 1967; Grice 1975).

Andererseits werden rechtsextreme Inhalte, die in den westeuropäischen Öffentlichkeiten seit 1945 verpönt bzw. mancherorts gesetzlich verboten sind (wie beispielsweise in Österreich durch das Verbotsgesetz seit 1947'13) (Engel und Wodak 2013), als Provokation mehr oder weniger explizit geäußert. Die intentionale Schamlosigkeit, die mit der Normalisierung bisher tabuisierter Inhalte und Verhaltensmuster einhergeht, schafft - so behaupte ich in Anlehnung an Thomas Scheffs Theorie zur Relevanz von Scham als Grundgefühl für Gruppenidentitäten - eine neue Gruppenkohäsion, festigt also die Gruppenidentität der WählerInnen rechtspopulistischer Parteien gegenüber den (oft moralisierenden) Eliten. Denn manche Wählerinnen und Wähler rechtspopulistischer Parteien betonen wiederholt, wie wichtig es ihnen sei, dass solche PolitikerInnen endlich aussprechen, was sie sich selbst schon immer gedacht haben, und sie sich somit ernst genommen fühlen (Wodak 2016, S. 141). Insofern könnte man von einer neuen ,Schamlosigkeit“" sprechen.

\section{Rechtsruck im „Post-Scham“-Zeitalter}

Rechtspopulistische Positionen sind, wie oben anhand der österreichischen Regierungskoalition beschrieben, in der sogenannten ,Mitte“ der Gesellschaft angekommen. Normalisierung funktioniert nicht über einzelne Wörter oder Metaphern, sondern ,ganze semantische Komplexe einschließlich ihrer Praxisbezüge“ (Link 2013, S. 15; Hervorhebung R. W.) verschieben sich. Konnotationen von Begriffen verändern sich, von eher positiven zu eher negativen Mitbedeutungen, und umgekehrt; Begriffe werden neu definiert und rekontextualisiert. Es können aber auch politische Forderungen, die zunächst von marginalisierten Gruppierungen vorgebracht werden, von Mainstream-Parteien aufgegriffen und umgesetzt werden. Dies ist ein durchaus gängiger Prozess, der häufig in Kämpfen um hegemoniale Bedeutungsmacht auftritt. Normalitäten ändern sich somit und werden dann auch akzeptiert (Wodak 2015b, S. 3).

Rheindorf und Wodak (2018) wiesen die Rekontextualisierung und Resemiotisierung rechtsextremer programmatischer Inhalte der FPÖ in den österreichischen politischen Mainstream nach: Einerseits handelt es sich um explizite Kontinuitäten (zum nationalsozialistischen Gedankengut), andererseits um kodierte, euphemistische Neuformulierungen, über diverse Politikfelder, inoffizielle und offizielle Parteiprogramme, Pamphlete, Wahlkampagnen (Plakate, Slogans, Reden) hinweg. Aufgrund der Kodierung werden rechtsextreme Inhalte für andere Wählergruppen

\footnotetext{
13 https://www.ris.bka.gv.at/GeltendeFassung.wxe?Abfrage=Bundesnormen\&Gesetzesnummer=10000207.
} Zugegriffen: 04.03.2017. 
akzeptabel. Durch eine Mehrebenenanalyse gelingt es, intertextuelle Verbindungen zwischen Parteipolitik und anderen gesellschaftlichen Bereichen aufzudecken, also die Übernahme rechtsextremer Ideologeme durch andere politische Parteien zu verdeutlichen.

Dieser Prozess lässt sich gut anhand von Johann Gudenus, früher FPÖ Vizebürgermeister von Wien (ohne Portefeuille) und seit Dezember 2017 Klubobmann der FPÖ im Parlament, nachvollziehen (Pollak 2015). So meint Gudenus: „Europa ist die Wiege der Weißen. Wir fordern eine europaweit abgestimmte Familien- und Bevölkerungspolitik, mit einem Bekenntnis dazu, dass Europa „weiß“ ist“"14; politische Gegner attackiert er u.a. mithilfe antisemitischer Stereotype: „Wenn man Rot und Grün zusammenmischt, erhält man gelb. Und gelb ist die Farbe von Judas, das ist die Farbe des Verrats!“15 Recht auf Asyl lehnt er grundsätzlich ab (,Asyl ist kein Menschenrecht" ${ }^{* 16}$ ) und vieles andere mehr. Sollte die FPÖ bei der nächsten Landtagswahl in Wien 2019 gewinnen, dann könnte Gudenus, Mitglied der schlagenden Verbindung Vandalia, Wiener Bürgermeister werden.

Gudenus' Überzeugungen ähneln dem Pamphlet Für ein freies Österreich (2013), geschrieben von FPÖ-Funktionär Michael Howanietz und herausgegeben von Norbert Hofer. Dieses wiederum weist viele intertextuelle Bezüge zum Handbuch freiheitlicher Politik (FPÖ-Bildungsinstitut 2013) auf, eine Richtlinie für Parteifunktionäre, mit Vorworten von H. C. Strache und Norbert Hofer, dem dritten Nationalratspräsidenten, der Van der Bellen im Kampf um die Präsidentschaft unterlag. Das obengenannte Pamphlet betont die wahre „Heimat“, die nur im ländlichen Raum zu finden sei, wo es höhere Geburtenraten, manuelle Arbeit und ,zeitlose Werte“ gibt (Howanietz 2013, S. 77). In diesem Kontext betont der Autor die biologischen und spirituellen Bezüge des „Volkes“, „de[n] Boden, aus dem unser Wasser kommt, in dem unsere Nahrung wächst, aus dem wir, wenigstens teilweise, unsere Energie beziehen, ist das heiligste Gut der Gemeinschaft" (Howanietz 2013, S. 141). Anklänge an eine nativistische „Blut-und-Boden“-Ideologie gehen damit einher: „Staaten und Völker sind in unserem Sprachgebrauch weitgehend Synonyme. Das Modell der Nation (von lat. nascere: geboren werden, entstehen) gründet sich auf der Abstammung der Bevölkerung“" (Howanietz 2013, S. 125).

In einer apokalyptischen Weltanschauung gelten Migranten als massive Bedrohung des ,,autochthonen, wahren“ Österreichers:

Die Zuwanderer aus jenen Ländern, die punktuell beginnen, über die ansässige Bevölkerung herzufallen [...] wissen um ihre Stärke. [...] Und auch deshalb ist der konfliktscheue, verwöhnte, überalternde, chronisch von tausenden Reizen überflutete und abgelenkte Mitteleuropäer auf bestem Wege zu einer bedrohten Spezies zu werden. [...] Denn sowohl Sprache und Kultur wie Bestand und Erhaltung der autochthonen Österreicher sind nachhaltig bedroht. (Howanietz 2013, S. 19-20)

\footnotetext{
14 Flugblatt des „Rings Freiheitlicher Jugend“ (Vorsitzender Johann Gudenus).

15 Johann Gudenus bei einer Wahlveranstaltung 2011 („Neue Freie Zeitung“; 27.10.2011).

16 Johann Gudenus, Pressemeldung 19.12.2014: www.ots.at/presseaussendung/OTS_20141219_OTS0076. Zugegriffen: 01.07.2017.
} 
Zur „Selbstzerstörung“ trägt, so wird behauptet, auch die - an sich selbstverständliche - Integration von Frauen in den Arbeitsmarkt bei (Howanietz 2013, S. 21). Legale Abtreibung wird abgelehnt (Howanietz 2013, S. 22). Eine anachronistische Genderpolitik propagiert ,[e]in evolutionäres Konzept, das sich an biologischen Gegebenheiten orientiert: der von keiner anderen Emotion überragbaren Mutter-KindBindung und des mütterlichen Brutpflegetriebes einerseits, sowie des Vaters als Versorger und Beschützer der Familie" (Howanietz 2013, S. 119).

Schuld für die drohende Apokalypse seien die Linken, Feministinnen, internationale Organisationen und die Medien, die metaphorisch als Instrumente einer angeblichen Massenvernichtung und als Krankheitserreger dargestellt werden:

Speziell das Massenmanipulationsinstrument Fernsehen ist deshalb in den Händen der Profithaie und nicht Volksinteressen vertretender Parteisoldaten die gefährlichste, die tödlichste „Massenvernichtungswaffe“. [...] Die höchste Gefahr einer infektiösen Ansteckung liegt heute im medialen Übertragungsweg. Hier pflanzt sich die Propaganda der herrschenden Klasse mit imposanter Geschwindigkeit und höchster Effektivität fort. (Howanietz 2013, S. 37)

Schließlich bietet das Pamphlet zwei Zukunftsalternativen an: Entweder würde das „wahre“ österreichische Volk langsam aussterben, ,indem wir jene, die uns ans Fell wollen, auch noch einladen, dies aus dem Inneren zu tun, wie die Wespenlarve die Made langsam von innen zerfrißt" (Howanietz 2013, S. 117); oder die derzeitigen Entwicklungen könnten in einem Bürgerkrieg enden (Howanietz 2013, S. 121). Damit werden Angst und Unsicherheit verstärkt. In jedem Fall „wird Europa dann brennen“ (Howanietz 2013, S. 113).

Natürlich vernimmt man solch rechtsextreme Rhetorik nicht in der Öffentlichkeit. Natürlich gibt es keine geradlinige oder gar kausale Verbindung zwischen dem Handbuch freiheitlicher Politik, dem Pamphlet Für ein freies Österreich, und den vielen Plakaten, Reden und TV-Debatten von FPÖ und der neuen ÖVP. Die Rekontextualisierung (und entsprechende Kodierung) erfolgen vielmehr über soziale Netzwerke, den Boulevard, Meinungsumfragen, einzelne Skandalisierungen und Provokationen, nachfolgende Leugnungen und Rechtfertigungen, und über häufig wiederholte Worthülsen (Wodak 2016, S. 38-40). Auf der Frontstage (Wodak 2011) äußern sich solche Ideologeme naturgemäß abgeschwächt, eingehüllt in ein Meer von Fahnen und Landschaftsbildern, die an Rieffenstahl'sche Ästhetik erinnern (Rheindorf und Wodak 2018).

Die neue ÖVP übernimmt nachweislich wesentliche Forderungen der FPÖ in Bezug auf Migrations- und Flüchtlingspolitik und propagiert demensprechend eine äußerst restriktive Einwanderungspolitik (Rheindorf und Wodak 2017), das Schließen der Grenzen (sogar nach Italien und Südtirol) und der Mittelmeerroute. Schamlos wurden und werden sowohl von der FPÖ wie auch der neuen ÖVP viele Gerüchte, Strohmannargumente und Falschmeldungen über Migranten und Flüchtlinge verbreitet, die allesamt zu einem einzigen Bedrohungsszenario verschmelzen, bestehend aus einer fantasierten „Invasion“ sogenannter „,illegaler Migranten“ (Ötsch und Horaczek 2017). Selbst ein ehrwürdiger ÖVP-Landeshauptmann, wie Wilfried Haslauer (Salzburg), übernimmt die Agenda der FPÖ, hinterfragt die Genfer Flüchtlingskon- 
vention und meint, ,[...] Asyl ist ein Grundrecht, ein theoretisches Gedankenspiel, das eine Grenze im Faktischen hat" ${ }^{\text {"17. }}$.

Um der Verpflichtung durch die Genfer Flüchtlingskonvention zu entkommen und einem weiteren Stimmenverlust an die FPÖ vorzubeugen, definieren ÖVP-PolitikerInnen Verfolgte und Flüchtende in ihrem Regierungsprogramm als ,illegale Migranten“ (Rheindorf und Wodak 2018). ${ }^{18}$ Damit wird unterstellt, diese seien gar nicht verfolgt, sondern Betrüger - Menschen, die sich als verfolgt ausgeben, um in reiche europäische Länder einzureisen, dort herrschende Sozialleistungen auszunutzen und derart den Wohlstand dieser Länder zu gefährden. Solche Trugschlüsse entfachen Ressentiment und Neid: Warum sollen denn Fremde in den Genuss von Leistungen kommen, die den Inländern etwas wegnehmen? Eine solche ausgrenzende und xenophobe - von der ehemals christlich-sozialen ÖVP getragene - Politik entspricht rechtspopulistischem Wohlfahrtschauvinismus und verdeutlicht die stete Normalisierung nach rechts. Nicht zufällig bezeichnen die Grünen den ÖVP Obmann Sebastian Kurz während des Wahlkampfes ,als den besseren Strache“19.

Funding Open access funding provided by University of Vienna.

Open Access Dieser Artikel wird unter der Creative Commons Namensnennung 4.0 International Lizenz (http://creativecommons.org/licenses/by/4.0/deed.de) veröffentlicht, welche die Nutzung, Vervielfältigung, Bearbeitung, Verbreitung und Wiedergabe in jeglichem Medium und Format erlaubt, sofern Sie den/die ursprünglichen Autor(en) und die Quelle ordnungsgemäß nennen, einen Link zur Creative Commons Lizenz beifügen und angeben, ob Änderungen vorgenommen wurden.

\section{Literatur}

Bettinger, Frank. 2007. Diskurse - Konstitutionsbedingung des Sozialen. In Foucaults Machtanalytik und soziale Arbeit: eine kritische Einführung und Bestandsaufnahme, Hrsg. Roland Anhorn, 75-90. Wiesbaden: Springer VS.

Betz, Hans-Georg. 2017. The new politics of resentment: radical right-wing populist parties in Western Europe. In The populist radical right. A reader, Hrsg. Cas Mudde, 338-351. London: Routledge.

Biskamp, Floris. 2017. On every level, by some means. Seven perspectives on and seven strategies against right-wing populism. Beitrag präsentiert bei Symposium: "Contesting Orbanism and Trumpism", Malmö, 16.11.2017.

Brubaker, Rogers. 2017. Why populism? Theory and Society https://doi.org/10.1007/s11186-017-9301-7.

Van de Cleen, Benjamin. 2017. Populism and nationalism. In Oxford handbook of populism, Hrsg. Cristóbal R. Kaltwasser, Paul Taggart. Oxford: Oxford University Press.

Engel, Jakob, und Ruth Wodak. 2013. "Calculated Ambivalence" and Holocaust Denial in Austria. In Analysing Fascist Discourse: European Fascism in Talk and Text, Hrsg. Ruth Wodak, John E. Richardson, 73-96. London: Routledge.

FPÖ-Bildungsinstitut. 2013. Handbuch freiheitlicher Politik. Ein Leitfaden für Führungsfunktionäre und Mandatsträger der Freiheitlichen Partei Österreichs, 4. Aufl., Wien: Freiheitliche Akademie. http:// www.fpoe-bildungsinstitut.at/documents/10180/13608/Handbuch_freiheitlicher_Politik+\%282\%29. pdf/3530ad0f-4bd0-47e2-9b8b-88a4b2a7a89d. Zugegriffen: 01. Nov. 2017.

\footnotetext{
17 http://www.salzburg.com/nachrichten/salzburg/politik/sn/artikel/haslauer-stellt- grundrecht-auf-asylinfrage-179009/. Zugegriffen: 05.03.2018.

$18 \mathrm{https} / / /$ kurier.at/politik/regierungsprogramm-rigorose-massnahmen-gegen-asylmissbrauch/302.354. 984. Zugegriffen: 05.03.2018.

19 http://www.oe24.at/oesterreich/politik/wahl2017/Im-Kern-ist-Kurz-ein-Strache/303871667. Zugegriffen: 05.03.2018.
} 
Goffman, Erving. 1967. Interaction ritual. Essays in face-to-face behavior. New Brunswick: Transaction Press.

Grabbe, Heather, und Stefan Lehner. 2017. Defending EU values in Poland and Hungary. Brüssel: Carnegie Europe.

Grice, Paul. 1975. Logic and conversation. In Speech acts, Hrsg. Peter Cole, Jerry L. Morgan, 41-58. New York: Academic Press.

Howanietz, Michael. 2013. Für ein freies Österreich. Souveränität als Zukunftsmodell. Wien: Freiheitlicher Parlamentsklub. Hrsg. Norbert Hofer.

Jäger, Margarete, und Siegfried Jäger. 2007. Deutungskämpfe: Theorie und Praxis kritischer Diskursanalyse. Wiesbaden: Springer VS.

Januschek, Franz, und Martin Reisigl (Hrsg.). 2014. Populismus in der digitalen Medienkultur. Osnabrücker Beiträge zur Sprachtheorie 86 (Special Issue).

Judt, Tony. 2007. Postwar: a history of Europe since 1945. London: Penguin.

Krastev, Ivan. 2017. Europadämmerung. Ein Essay. Frankfurt: Suhrkamp.

Krasteva, Anna. 2017. Re/de/constructing the far-right youth: between the lost generation and the contestatory citizenship. In Understanding the populist shift: othering in a Europe in crisis, Hrsg. Gabriela Lazaridis, Gianni Campani, 150-178. London: Routledge.

Kriesi, Hanspeter, und Takis S. Pappas (Hrsg.). 2015. European populism in the shadow of the great recession. Colchester: ECPR Press.

Laclau, Ernesto. 2005. On populist reason. London: Verso.

Lakoff, George. 2004. Don't think of an elephant: know your values and frame the debate. White River Junction: Chelsea Green.

Link, Jürgen. 2013. Versuch über den Normalismus: wie Normalität produziert wird, 3. Aufl., Göttingen: Vandenhoeck \& Ruprecht.

Manoschek, Walter. 2002. FPÖ, ÖVP, and Austria's Nazi Past. In The Haider phenomenon in Austria, Hrsg. Ruth Wodak, Anton Pelinka, 3-16. New Brunswick: Transaction.

Moffitt, Benjamin. 2017. The global rise of populism. Performance, political style, and representation. Stanford: Stanford University Press.

Montgomery, Martin. 2017. Post-truth politics? Authenticity, populism and the electoral discourses of Donald Trump. Journal of Language and Politics 16(4):619-639.

Mudde, Cas, und Cristobal R. Kaltwasser. 2017. Populism. Oxford: Oxford University Press.

Ötsch, Walter, und Nina Horaczek. 2017. Populismus für Anfänger: Anleitung zur Volksverführung. Frankfurt am Main: Westend.

Pajnik, Mojca, und Birgit Sauer (Hrsg.). 2017. Populism and the Web: communicative practices of parties and movements in Europe. London: Ashgate.

Pelinka, Anton. 2013. Right-wing populism: concept and typology. In Right-wing populism in Europe. Politics and discourse, Hrsg. Ruth Wodak, Majid KhosraviNik, und Brigitte Mral, 3-22. London: Bloomsbury.

Pels, Dick. 2012. The new national individualism - populism is here to stay. In Populism in Europe, Hrsg. Eric Meijers, 25-46. Linz: Planet.

Pfahl-Traughber, Armin. 2015. Die Nicht-Erkennung des NSU-Terrorismus. In Jahrbuch Extremismus und Demokratie 27, Hrsg. Uwe Backes, Alexander Eckhard, und Jesse Gallus, 73-96. Baden-Baden: Nomos.

Pollak, Alexander. 2015. Der Hassprediger. Der aufhaltsame Aufstieg des Johannes G. Berlin: Epubli.

Priester, Karin. 2007. Populismus. Historische und aktuelle Erscheinungsformen. Frankfurt am Main: Campus.

Rauscher, Hans. 2017. „Stille Machtergreifung“ der Burschenschafter, Der Standard. http://derstan dard.at/2000063481709/Stille-Machtergreifung-der-Burschenschafter (Erstellt: 1. Sept. 2017). Zugegriffen: 15. Sept. 2017.

Rheindorf, Markus. 2017. Integration durch Strafe? Die Normalisierung paternalistischer Denkfiguren zur „Integrationsunwilligkeit“. Zeitschrift für Diskursforschung 5(2):182-206.

Rheindorf, Markus, und Ruth Wodak. 2017. Borders, fences and limits: protecting Austria from refugees. Metadiscursive negotiation of meaning in the current refugee crisis. Journal Immigrant \& Refugee Studies https://doi.org/10.1080/15562948.2017.1302032.

Rheindorf, Markus, und Ruth Wodak. 2018. "Austria First" revisited: a diachronic cross-sectional analysis of the gender and body politics of the extreme right. Patterns Prejudice (in Druck).

Rydgren, Jens (Hrsg.). 2017. Handbook of the radical right. Oxford: Oxford University Press.

Scharsach, Hans-Hennig. 2017. Stille Machtergreifung. Hofer, Strache und die Burschenschaften. Wien: $\mathrm{K} \& \mathrm{~S}$. 
Scheff, Thomas. 2000. Shame and the social bond: a sociological theory. Sociological Theory 18(1):84-99. Stavrakakis, Yannis. 2017. Discourse theory in populism research: three challenges and a dilemma. Journal Language and Politics 16(4):523-534.

Wodak, Ruth. 2011. The discourse of politics in action. Politics as usual. Basingstoke: Palgrave.

Wodak, Ruth. 2015a. Politics of fear: what right-wing populist discourses mean. London: Sage.

Wodak, Ruth. 2015b. „Normalisierung nach Rechts“: Politischer Diskurs im Spannungsfeld von Neoliberalismus, Populismus und Kritischer Öffentlichkeit. Linguistik Online 73(4):27-44.

Wodak, Ruth. 2016. Politik mit der Angst. Zur Wirkung rechtspopulistischer Diskurse. Wien: Konturen.

Wodak, Ruth. 2017. The establishment, the elites, and the people: who's who? Journal Language and Politics 16(4):551-565. https://doi.org/10.1075/jlp.17030.wod.

Wodak, Ruth, und Anton Pelinka (Hrsg.). 2002. The Haider Phenomenon in Austria. New Brunswick: Transaction.

Wodak, Ruth, und Markus Rheindorf. 2018. The Austrian Freedom Party. In The new authoritarianism: a risk analysis of the Alt-right phenomenon, Hrsg. Alan Waring. New York: Ibidem. 